\title{
Prevalencia de microorganismos y perfil de resistencia antimicrobiana en bovinos lecheros de Paraguay
}

\author{
Pedrozo, P.R.'; Torres, Ñ.M. ${ }^{2}$; López, R.D. ${ }^{3}$; Alonso, V.N.4; Valiente, O.L. ${ }^{5}$ \\ Univ.Nac.Asunción, Fac.Cs.Vet., San Lorenzo, Paraguay. \\ ${ }^{1}$ Dpto.Patol.\& Clín., ${ }^{2}$ Dpto.Cs.Fisiol., ${ }^{3}$ Dpto.Microb.Inmunol., \\ ${ }^{4}$ Dpto.Balanceados, ${ }^{5}$ Dpto.Bromatol. E-mail: ovaliente $\operatorname{a}$ vet.una.py
}

\begin{abstract}
Resumen
Pedrozo, P.R.; Torres, Ñ.M.; López, R.D.; Alonso, V.N.; Valiente, O.L. Prevalencia de microorganismos y perfil de resistencia antimicrobiana en bovinos lecheros de Paraguay. Rev. Vet. 32: 1, 25-30, 2021. La mastitis bovina es la inflamación de las glándulas mamarias, causada mayoritariamente por invasión de microorganismos patógenos. El objetivo del estudio fue identificar los microorganismos más frecuentemente aislados y determinar el perfil de resistencia antimicrobiana en función de las especies o grupo de especies de microorganismos aislados. Se tomaron 411 muestras de leche de igual números de vacas de cuartos positivos al California Mastitis Test (CMT), de razas lecheras de 3 departamentos de Paraguay (224, 137 y 50 muestras), que se sometieron a cultivos microbianos de identificación fenotípica de especies. La sensibilidad microbiana a antimicrobianos se evaluó por dilución en placa. Las frecuencias absoluta y relativa de microorganismos, así como las resistencias, se cuantificaron con el software Epiinfo 7.0. El 37,4\% de los microorganismos correspondieron a Streptocccus sp, el 35,3\% al grupo Staphylococcus coagulasa negativos (SCN) y el 16,1\% a los Staphylococcus coagulasa positivos (SCP). En orden decreciente, los perfiles de resistencia antimicrobiana fueron: tetraciclina, penicilina, sulfatrimoprim, rifampicina y ampicilina $(34,7 ; 19,7 ; 14,4 ; 8,2$ y 6,4\%, respectivamente). Los Streptocccus spp fueron resistentes a 7/10 (rango de 51,3 a $1,7 \%$ ), los SCN a $8 / 10$ (rango de 29,7 a $2 \%$ ) y los SCP a $7 / 10$ (rango de 44,7 a $2,1 \%$ ), mientras que las enterobacterias fueron resistentes a $7 / 20$, fundamentalmente a la ampicilina (4/5). También se observaron multiresistencias, aunque en un número pequeño. Se concluye que los grupos de microorganismos más frecuentes fueron los Streptocccus $s p$, seguidos de los SCN y en tercer lugar los SCP. Las resistencias antimicrobianas, de mayor a menor, fueron: tetraciclina, penicilina y sulfatrimetoprim, siendo todavía poco frecuente la multiresistencia.
\end{abstract}

Palabras clave: bovinos, mastitis, aislamiento microbiano, resistencia antimicrobiana.

\begin{abstract}
Pedrozo, P.R.; Torres, Ñ.M.; López, R.D.; Alonso, V.N.; Valiente, O.L. Prevalence of microorganisms and antimicrobial resistance profile in dairy cattle of Paraguay. Rev. Vet. 32: $1,25-30,2021$. The bovine mastitis is an inflammation of the mammary glands of endemic presentation mainly caused by the invasion of pathogenic microorganisms. The objective of the study was to identify the most frequently isolated microorganisms and to determine the antimicrobial resistance profile according to the species or group of species of microorganisms isolated. The 411 milk samples were taken from equal numbers of California Mastitis Test CMT-positive quarter cows from dairy breeds in 3 departments of Paraguay $(224,137$ and 50 from the Central, Cordillera and Paraguari departments), which were subjected to microbial species identification cultures. Microbial sensitivity to antimicrobials was evaluated by plate dilution. The absolute and relative frequency of microorganisms and resistances were quantified with the Epiinfo 7.0 software. The $37.4 \%$ of the microorganisms corresponded to Streptocccus sp, $35.3 \%$ to the coagulase negative Staphylococcus group (SCN) and $16.1 \%$ to the coagulase positive Staphylococcus (SCP) as the most frequent. The antimicrobial resistance profile in decreasing order was tetracycline, penicillin, sulfatrimoprim, rifampicin and ampicillin $(34.7 ; 19.7 ; 14.4 ; 8.2$ and $6.4 \%$, respectively). Streptocccus sp were resistant to $7 / 10$ (range 51.3 to $1.7 \%$ ), SCN to $8 / 10$ (range 29.7 to $2 \%$ ), and SCP to $7 / 10$ (range 44.7 to $2.1 \%$ ), while enterobacteria were resistant to $7 / 20$, mainly to ampicillin (4/5). Multiresistance was also observed, although in a small number. It was concluded that the most frequent groups of microorganisms were Streptocccus sp, followed by SCN and thirdly SCP. Antimicrobial
\end{abstract}


resistance, from highest to lowest, was to tetracycline, penicillin and sulfatrimetoprim, being still rare multiresistance.

Key words: bovine, mastitis, microbial isolation, antimicrobial resistance.

\section{INTRODUCCIÓN}

La mastitis bovina es la inflamación de las glándulas mamarias de las vacas lecheras, causadas por la invasión y destrucción de los tejidos productores de leche por microorganismos patógenos ${ }^{24}$. Las bacterias responsables de causar mastitis incluyen tanto grampositivas como gramnegativas (Staphylococcus, Streptococcus, Escherichia coli y Klebsiella pneumoniae $\left.{ }^{6}\right)$. Los Staphylococcus coagulasa negativos son patógenos oportunistas que también han surgido como importantes patógenos de mastitis ${ }^{27}$.

La mastitis es una enfermedad de presentación endémica y es considerada una de las más costosas en la industria láctea. Puede presentarse de forma clínica o subclínica y las consecuencias económicas pueden estar dadas por el tratamiento, las pérdidas en la producción, el descarte temprano de animales, los cambios en la calidad de la leche y el riesgo de otras enfermedades ${ }^{13,28}$.

Los antimicrobianos se utilizan con frecuencia en las terapias para el control de varias bacterias que causan mastitis en las granjas lecheras ${ }^{23}$. Sin embargo, el tratamiento de la mastitis, así como su control, es difícil ${ }^{1}$. La aparición de las bacterias resistentes a los antimicrobianos es una de las razones de la baja tasa de recuperación de la mastitis ${ }^{11}$.

Actualmente, la resistencia a los antimicrobianos se considera un problema de salud pública en todo el mundo ${ }^{19}$. Las bacterias multi-resistentes a las drogas están muy extendidas, debido a la presión selectiva resultante del uso extensivo de antimicrobianos (ATM) en la medicina veterinaria y humana, y debido a la adquisición de determinantes de resistencia antimicrobiana de otras bacterias ${ }^{16}$.

En Paraguay el uso de ATM en el tratamiento de la mastitis bovina no está regulado y se permite el acceso libre a los mismos, en ocasiones sin supervisión técnica, lo que se considera puede favorecer la aparición de cepas con resistencia a dichos agentes.

Resulta importante, en primer lugar, identificar las especies microbianas más prevalentes causantes de mastitis infecciosas en una zona o región de producción. En segundo lugar, identificar los niveles de resistencias a los antimicrobianos (RAM) que presentan, de tal manera a realizar un uso más efectivo y criterioso de los ATM. La RAM es una realidad en el mundo y según la OMS resulta en una de las mayores amenazas para la salud, la seguridad alimentaria y el desarrollo de los países ${ }^{19}$.

En este sentido, en Paraguay hay pocos estudios publicados que se hayan enfocado en la RAM ${ }^{10}$, teniendo como antecedente el perfil de resistencia in vi- tro a ATM de cepas causantes de mastitis a nivel de establecimientos de pequeña y mediana producción, pero hasta donde se sabe no se realizó ningún estudio que abarque a establecimientos de los 3 niveles de producción (pequeño, mediano y grande) y que haya abarcado a 3 departamentos del país en donde se concentra la producción de leche proveída al área metropolitana de la gran Asunción.

El objetivo de este estudio fue identificar los microorganismos más frecuentemente aislados en leche de cuartos mamarios positivos al California Mastitis Test (CMT) y determinar el perfil de resistencia a antimicrobianos en función al grupo o especies de agentes microbianos aislados.

\section{MATERIAL Y MÉTODOS}

Lugar de estudio. Se tomaron 411 muestras de leche de la misma cantidad de vacas lecheras, cuyos cuartos dieron positivo a CMT de los departamentos Central ( 8 tambos, $n=224)$, Cordillera ( 6 tambos, $n=$ 137) y Paraguarí ( 3 tambos, $n=50$ ), en su mayoría de razas Holando, Jersey y cruzas Holando-Jersey. El periodo de lactación, la cantidad de leche producida, así como los sistemas de producción fueron diferentes.

Aspectos éticos. La toma de la muestra se realizó durante el ordeño de la mañana o de la tarde. Dado que no se practicaron procedimientos experimentales; no fue necesario solicitar el aval de un comité de ética. El estudio contó con el consentimiento informado de los productores para la toma de las muestras. Esta toma fue realizada por investigadores del proyecto y estudiantes de último año de la carrera de ciencias veterinarias de la Universidad Nacional de Asunción, quienes recibieron un entrenamiento previo para la realización del procedimiento de la prueba CMT, la conservación y el envío de muestras al laboratorio cumplieron los parámetros establecidos por el National Mastitis Council ${ }^{18}$.

Prueba de CMT. Se realizó en 1644 cuartos en producción.. De los cuartos mamarios que presentaron un resultado mayor o igual a trazas, se procedió a la toma de aproximadamente $20 \mathrm{ml}$ de muestra (colocados en frascos estériles), haciendo un poolcuando más de un cuarto de una misma vaca fue positivo para al CMT. Estas muestras fueron refrigeradas a $5^{\circ} \mathrm{C}$ y antes de las 24 horas se hicieron llegar al Laboratorio de Microbiología de la Facultad de Ciencias Veterinarias de la Universidad Nacional de Asunción.

Cultivo bacteriológico y tipificación bacteriana. Las muestras de leche, fueron sembradas en agar san- 
gre, agar MacConkey y agar Tripticasa Soya, incubándose entre 18 y 24 horas. En las cajas en las que no hubo crecimiento volvieron a ser incubadas por 24 horas, posteriormente fue realizada la coloración de Gram para determinar morfología y tinción clasificándolas como cocos o bacilos Gram positivos y Gram negativos. A los cocos Gram positivos se les realizó la prueba de la catalasa, y a los negativos se les implementó la prueba de la coagulasa, clasificando los cocos como coagulasa positivos o negativos. Posteriormente, esta metodología se complementó con un sistema de identificación comercial, confirmando género y especie; de esta forma se pudo discriminar entre Staphilococcus aureus de otros estafilococos coagulasa positivos como Staphilococcus intermedius y Staphilococcus hyicus. Los cocos Gram positivos y catalasa negativos se identificaron como Streptococcus sp a los cuales se les efectuó la prueba de CAMP, hidrólisis de la esculina, hipurato e inulina y crecimiento en $\mathrm{NaCl}$. A los bacilos Gram negativos se les implementó las pruebas de oxidasa, indol, triple azúcar, RM-VP, LIA, citrato y urea, para determinar género y especie ${ }^{15}$. La evaluación de la sensibilidad bacteriana a los antimicrobianos se realizó mediante el método de dilución en placa, siguiendo las recomendaciones del National Commitee for Clinical Laboratory Standards (CLSI) ${ }^{5}$. Todos los ATM se evaluaron como drogas puras con potencia conocida, los cuales se mantuvieron refrigeradas entre 4- $8^{\circ} \mathrm{C}$ por un máximo de un mes. Los ATM ensayados fueron aquellos de uso más frecuente para diferentes enfermedades en los rebaños lecheros del país y se seleccionaron para las diferentes especies bacterianas de acuerdo a su espectro de acción. Las drogas utilizadas fueron: oxacilina, penicilina, eritromicina, clindamicina, cloranfenicol, tetraciclina, ampicilina, cefotaxima, gentamicina, trimetropimsulfametoxazole, rifampicina, ciprofloxacina, cefoxitin, ceftazidima, cefixima, amoxicilina, ácido clavulánico, cefepime, ceftriaxona, ácido nalidixico, imipenem, ertapenem, aztreonam, cefoprezona sulbactam, amikamicina, y cefuroxima. Para el análisis de los resultados, se consideró que cada cepa bacteriana se comportaba individualmente frente a un antimicrobiano determinado, la sensibilidad / resistencia se basó en las recomendaciones del CLSI $2013^{5}$.

Diseño metodológico. El muestreo se efectuó por conveniencia y de manera transversal de los 17 tambos de los 3 departamentos de Paraguay, utilizándose los registros de tambos del organismo oficial de salud animal (SENACSA).

Análisis estadísticos. Se realizaron mediante estadísticas descriptivas (determinación de frecuencias absoluta y relativa de los datos), utilizando el software estadístico Epiinfo 7.0, evaluando prevalencia microbiana y de resistencia antimicrobiana, así como el cruzamiento entre estas dos variables.
Tabla 1. Frecuencia de patógenos microbianos aislados de leche positiva a CMT (286 microorganismos aislados en 411 muestras, sin crecimiento en 159), en los Departamentos Central, Cordillera y Paraguarí (Paraguay).

\begin{tabular}{lcc}
\hline especies microbianas & $\mathrm{N}^{\circ}$ frec. & \% frec. \\
\hline Streptococcus dysgalactiae & 79 & 27,6 \\
Staphylococcus epidermidis & 55 & 19,2 \\
Staphylococcus aureus & 28 & 9,8 \\
Streptococcus mutans & 12 & 4,2 \\
Corynebacterium sp & 12 & 4,2 \\
Streptococcus agalactiae & 11 & 3,8 \\
Otros SCN & 46 & 16,1 \\
Otros SCP & 19 & 6,6 \\
Otros Streptococcus & 17 & 5,9 \\
Klebsiella pneumoniae & 3 & 1 \\
Escherichia coli & 2 & 0,7 \\
Enterococcus canis & 1 & 0,3 \\
Levadura & 1 & 0,3 \\
\hline total & 286 & 100 \\
\hline
\end{tabular}

\section{RESULTADOS Y DISCUSIÓN}

En la Tabla 1 se pudo observar que el 35,7\% (102/286) de los microorganismos aislados correspondieron a 3 especies de Streptococcus ( $S$. dysgalactiae, S. mutans, S. agalactiae) y a otros Streptococcus (17/286) como S. equi, uberis, mitis, porcinus y pneumonie. El 35,3\% (101/286) fueron del grupo SCN de las siguientes especies Staphylococcus epidermidis (55), S. saprophyticus (8), S. capitis (8), S. simulans (7), $S$. shleiferi (6), S. lugdunensis (6), S haemolyticus (6) y $S$. warneri (5). Los SCP representaron el 16,4\% (47/286) que correspondieron a $S$. aureus (28), S. hyicus (7), $S$. intermedius (7), S. pseudointermedius (4) y S. schleifericoagulans (1). Se aislaron en menor número: Klebsiella pneumoniae, Escherichia coli, Enterococcus canis y levadura.

Los microorganismos causantes de mastistis fueron clasificados de acuerdo a su hábitat, interacción con el pezón y patogenicidad, como microorganismos contagiosos, ambientales y otros microorganismos ${ }^{2}$. En el presente estudio el más frecuente fue un microorganismo ambiental (el Streptococcus dysgalactiae), seguido por un microorganismo oportunista (S. epidermidis), siendo también frecuentes dos agentes causales de mastitis contagiosa como el $S$. aureus y el $S$. agalactiae, datos que concuerdan con otros investigadores ${ }^{4}$. Cabe señalar que en la actualidad los SCN son considerados causantes de mastitis emergentes en el mundo ${ }^{22}$.

El perfil de la resistencia a los ATM en los departamentos Central Cordillera y Paraguarí (Tabla 2) fue más frecuente en 5 de éstos. En orden decreciente fueron: tetraciclina, penicilina, sulfatrimetoprim, rifampicina y ampicilina (variando desde $35 \%$ para la tetraciclina hasta poco más de $6 \%$ para la ampicilina). También apareció resistencia en menor frecuencia a la eritromicina, clindamicina, gentamicina, oxacilina, 
Tabla 2. Perfil de resistencia a los antimicrobianos en los Departamentos Central, Cordillera y Paraguarí (Paraguay).

\begin{tabular}{lccc}
\hline antimicrobiano & $\mathrm{N}^{\circ}$ frec & \% frec & $\mathrm{N}$ \\
\hline Tetraciclina & 99 & 34,7 & 285 \\
Penicilina & 55 & 19,7 & 279 \\
Sulfatrimetoprim & 41 & 14,4 & 285 \\
Rifampicina & 23 & 8,2 & 280 \\
Ampicilina & 8 & 6,4 & 125 \\
Eritromicina & 11 & 3,9 & 285 \\
Clindamicina & 7 & 2,5 & 279 \\
Gentamicina & 4 & 2,4 & 166 \\
Oxacilina & 4 & 1,8 & 219 \\
Cloranfenicol & 4 & 1,4 & 285 \\
Ciprofloxacina & 1 & 0,6 & 166 \\
\hline
\end{tabular}

cloranfenicol y ciprofloxacina, que en todos los casos fue inferior al $4 \%$.

La alta resistencia a la tetraciclina observada pudo deberse a la utilización masiva de este antimicrobiano motivado por el costo asequible que presenta, que fue marcadamente inferior a lo reportado por un estudio francés (próximos a 7\%) durante 10 años de evaluación, mostrando una tendencia estacionaria ${ }^{3}$, aunque el trabajo fue realizado solo en SCP.

Sin embargo, en un reporte colombiano se encontró una alta prevalencia de resistencia a la tetraciclina (58\%) en el grupo de SCN aislados por cultivo microbiológico ${ }^{22}$. El siguiente antimicrobiano con frecuencia de resistencia importante fue la penicilina, probablemente por pertenecer al grupo de los antibióticos más utilizados en los casos de mastitis bovina ${ }^{4}$.

En un estudio europeo realizado en leche de cuartos mamarios con mastitis clínica se reportaron niveles de resistencia variable de 25,1 y $29,1 \%{ }^{9}$, que incluso, fueron superiores a lo observado en el presente estudio, explicado probablemente por el grupo de microorganismos en que se realizó (S. aureus y SCN) ${ }^{9}$. No obs- tante, en otro estudio realizado en Colombia en SCN aislados por cultivo microbiológico, la resistencia fue incluso mucho más elevada (61\%) ${ }^{22}$.

La resistencia de las diferentes especies microbianas a los distintos ATM se comportó de la siguiente manera: de los 10 probados para el grupo de los Streptococcus sp, en 7 de ellos se encontró resistencia (variación de 1,7 a $51,3 \%$ ), con las frecuencias más altas para tetraciclina, sulfatrimetoprim y rifampicina (Tabla 3), mientras que los SCN presentaron resistencia a $8 / 10$ antimicrobianos (variación de 2 a 29,7\%), siendo las mayores resistencias a penicilina (30/101), tetraciclina $(19 / 101)$ y eritromicina $(5 / 101)$.

Los SCP 7/10 ATM presentaron algún grado de resistencia (rango de 2,1 a 44,7\%), observándose las mayores resistencias a la penicilina (21/47) seguida de tetraciclina (17/47). El Corynebacterium $s p$ solo fue resistente a un ATM a la sulfatrimetoprim. En el caso de las enterobacterias se probaron en 20 ATM según las sugerencias de CLSI ${ }^{5}$, con resistencia a 7 de éstos, siendo la más importante la ampicilina ( $1 \mathrm{E}$. coli y 3 Klebsiella), seguida por la sulfatrimetoprim (2/5: 1 por cada especie bacteriana citada) y la tetraciclina (2/5: ambos para la $E$. coli).

En el presente estudio la resistencia a la tetraciclina de los Streptococcus fue muy alta (51,3\%), concordante con los $59 \%$ de prevalencia de resistencia ATM reportada en tambos grandes de China ${ }^{7}$. Dentro de este grupo de especies la resistencia ATM del $S$. dysgalactiae más relevante fue a la tetraciclina $(54,4 \%)$, incluso más alta a los 33\% (de 1180 muestras reportadas en 2018 en vacas con mastitis clínica de China ${ }^{29}$ )

Respecto a la resistencia de los Streptococcus $s p$ a la penicilina y ampicilina, si bien fue baja $(3,4 \%$ y $2,5 \%$ ), en principio no debería existir resistencia a este antimicrobiano. En tal sentido, se ha reportado ${ }^{21}$ que los antimicrobianos como penicilina, amoxicilina-clavulamico, cefazolina, rifampicina, cloranfenicol y vancomicina, muestran muy buena eficacia in vitro contra

Tabla 3. Perfil de resistencia a diversos antimicrobianos de Streptococcus sp, SCN, SCP, Cory nebacterium y enterobacterias en muestras de leche positivas a CMT.

\begin{tabular}{|c|c|c|c|c|c|c|c|c|c|c|}
\hline \multirow[t]{2}{*}{ antimicrobianos } & \multicolumn{2}{|c|}{$\begin{array}{c}(\mathrm{N}=119) \\
\text { Streptococcus }\end{array}$} & \multicolumn{2}{|c|}{$\begin{array}{c}(\mathrm{N}=101) \\
\mathrm{SCN}\end{array}$} & \multicolumn{2}{|c|}{$\begin{array}{c}(\mathrm{N}=47) \\
\mathrm{SCP}\end{array}$} & \multicolumn{2}{|c|}{$\begin{array}{c}(\mathrm{N}=12) \\
\text { Corynebacterium }\end{array}$} & \multicolumn{2}{|c|}{$\begin{array}{c}(\mathrm{N}=5) \\
\text { Enterobacterias }\end{array}$} \\
\hline & $\mathrm{N}^{\circ}$ & $\%$ & $\mathrm{~N}^{\circ}$ & $\%$ & $\mathrm{~N}^{\circ}$ & $\%$ & $\mathrm{~N}^{\circ}$ & $\%$ & $\mathrm{~N}^{\circ}$ & $\%$ \\
\hline Tetraciclina & 61 & 51,3 & 19 & 18,8 & 17 & 36,2 & -- & & 2 & 40 \\
\hline Penicilina & 4 & 3,4 & 30 & 29,7 & 21 & 44,7 & -- & & - & \\
\hline Sulfatrimetoprim & 32 & 26,9 & 2 & 2 & -- & & 5 & 41,7 & 2 & 40 \\
\hline Rifampicina & 20 & 16,8 & 2 & 2 & 1 & 2,1 & -- & & 1 & 20 \\
\hline Ampicilina & 3 & 2,5 & - & & - & & -- & & 4 & 80 \\
\hline Eritromicina & 3 & 2,5 & 5 & 5 & 2 & 4,3 & -- & & 1 & 20 \\
\hline Clindamicina & 2 & 1,7 & 3 & 3 & 2 & 4,3 & -- & & -- & \\
\hline Gentamicina & -- & & 3 & 3 & 1 & 2,1 & -- & & & \\
\hline Oxacilina & -- & & 3 & 3 & -- & & -- & & & \\
\hline Cefoxitina & -- & & & & 2 & 4,3 & -- & & -- & \\
\hline Cefepime & & & & & & & & & 1 & 20 \\
\hline Cefuroxime & & & & & & & & & 1 & 20 \\
\hline
\end{tabular}


el grupo de Streptococcus ( $S$. agalactiae, $S$ dysgalactiae, S. uberis), aislados de bovinos con mastitis subclínica, por lo que estos tipos de ATM son frecuentemente usados para el tratamiento contra S. agalactiae ${ }^{8}$, aunque en el presente estudio la rifampicina evidenció un nivel de resistencia de $16,8 \%$.

La prevalencia de la resistencia a la sulfatrimetoprim fue de $26,9 \%$, muy inferior a lo informado por investigadores ${ }^{29}$ para las sulfonamidas $(83 \%)$, aunque superior al $17 \%$ encontrado por otros ${ }^{17}$ para $S$. dysgalactiae aislados en un estudio. Se ha descripto una resistencia intrínseca para la mayoría de los Streptococcus a las sulfonamidas ${ }^{26}$.

Para el grupo de $\mathrm{SCN}$, la resistencia a penicilina y tetraciclina ( 30 y $19 \%$, respectivamente) fue importante en este estudio, que resultó inferior a lo reportado en un trabajo colombiano para la penicilina $(38,9 \%)$, pero superior a los $8,3 \%$ a la tetraciclina ${ }^{14}$. En tal estudio se reportó una mayor resistencia a la eritromicina $(13,9 \%$ vs $5 \%)^{14}$.

En otra investigación realizada en el mismo país se encontraron prevalencias de resistencias mucho más altas a las del presente estudio, que fueron de 61,58 y $42 \%$ para penicilina, tetraciclina y eritromicina respectivamente ${ }^{22}$. También se observó $3 \%$ de meticilino resistencia (oxacilina) lo que concuerda con los 3,09\% hallados por otros ${ }^{25}$, aunque en un trabajo publicado recientemente no observó resistencia a la oxacilina ${ }^{14}$.

Dentro de los SCP el microorganismo aislado en mayor número fue el Staphylococcus aureus (28/47), en el que se observaron las mayores resistencias de 53,6 y $50 \%$ a la penicilina y tetraciclina respectivamente. Algunos estudios ${ }^{14,25}$ mostraron una menor resistencia a la penicilina $(23,1 \%$ y $33,85 \%)$, no obstante en otros trabajos se reportó un mayor valor de resistencia $(66 \%)^{7}$.

Para la tetraciclina también se reportan menores niveles de resistencia a ATM como $18,5 \%{ }^{14}$ y $18 \%{ }^{7}$. Se observó un caso $(3,6 \%)$ de meticilino resistencia a cefoxitina, que fue similar a los $4,6 \%$ reportados por otros ${ }^{14}$ para la misma droga, e igual número de resistencia para eritromicina y clindamicina $(1 / 28)$ en este trabajo, que fue mucho menor a la resistencia reportada por otros ${ }^{14}$ para estos dos antimicrobianos $(15,4 \%$ en ambos ATM).

La resistencia a Corynebacterium sp solo se evidenció para sulfatrimetoprim (5/12), no habiendo resistencia a penicilina ni tetraciclina, lo cual coincide con lo resultado de otras investigaciones ${ }^{9}$, en el sentido de no reportar resistencia a penicilina y tetraciclina, a pesar de que ellos no hicieron la prueba a sulfonamidas.

La mayor resistencia observada en enterobacterias fue para la ampicilina (4/5), seguida de sulfatrimetoprim y tetraciclina ( $2 / 5$ en ambos ATM). La resistencia para la ampicilina fue mayor en Klebsiella (3/3) que para $E$. coli $(1 / 2)$, superior a las resistencias a los mismos ATM ( $78,6 \%$ y $24 \%$, respectivamente) reportadas por otros ${ }^{9}$.
Se debe tener presente el alto rango de resistencia esperado en este género bacteriano a causa de la baja susceptibilidad inherente a la ampicilina ${ }^{9}$. En el caso de la tetraciclina ambas resistencias fueron para $E$. coli (2/2), muy superior a lo reportado para esta especie $(23,6 \%)^{7,9}$, con la reserva debido al número reducido de enterobacterias aisladas en el presente estudio.

Se evidenció multi-resistencia ( $>$ a 2 ATM) en 3 cepas (3/79) de $S$. dysgalactiae que fue la especie aislada en mayor número (79/119), evidenciando resistencia a la tetraciclina, sulfatrimetoprim y rifampicina. La multi-resistencia observada en este estudio fue muy inferior (21/101) a la reportada ${ }^{7}$, aunque fueron a diferentes tipos ATM de los observados en el presente estudio, a excepción de la tetraciclina.

Los SCN presentaron 6/101 cepas multi-resistentes, todas fueron resistentes a la penicilina, 3 a oxacilina, 4 a eritromicina, 3 a gentamicina, 2 a tetraciclina y rifampicina, y 1 sola cepa a trimetroprim sulfametoxazo1e. Además, una cepa de Staphylococcus schleiferi fue resistente a 7 ATM. Estos niveles de multi-resistencia fueron mucho más bajos que los informados por Raspanti para el S. haemolyticus (42,8\%) en Argentina ${ }^{20} \mathrm{y}$ para SCN $(78,6 \%)$ por Gizaw en Etiopía ${ }^{12}$.

Para los SCP se encontraron 4/47 cepas multi-resistentes, más frecuentemente en Staphylococcus aureus. Todas ellas fueron resistentes a penicilina, 3 a meticilina y 2 a clindamicina y eritromicina (una sola cepa a gentamicina, tetraciclina y rifampicina). También una cepa de Staphylococcus intermedius fue resistente a 6 ATM, esta multi-resistencia observada ${ }^{10}$ concuerda con lo reportado anteriormente ${ }^{25}$, aunque es inferior al $33 \%$ reportado otros ${ }^{7}$, aunque concuerdan en su patrón de resistencia con los siguientes antimicrobianos: penicilina, oxacilina, clindamicina y tetraciclina.

Los microorganismos más prevalentes en las 17 fincas lecheras de los 3 departamentos de Paraguay, donde se tomaron las muestras, fueron especies propias del medio ambiente como el Streptococcus dysgalactiae, del grupo SCN como el Staphylococcus epidermidis y uno de los patógenos más importantes causantes de mastitis contagiosa, el Staphylococcus aureus.

Los Streptococcus sp fueron muy resistentes a la tetraciclina $(51,3 \%)$. Los $\mathrm{SCN}$ fueron más resistentes a la penicilina $(29,7 \%)$ al igual que los SCP $(44,7 \%)$. Los Corynebacterium fueron resistente a la sulfatrimetoprim $(41,7 \%)$, en tanto, que las enterobacterias fueron altamente resistentes a la ampicilina (4/5). La multiresistencia observada en este trabajo fue baja (3/119) en Streptococcoccus, (6/101) en SCN y (4/47) en SCP, pero llama la atención que todos los multi-resistentes $\mathrm{SCN}$ y SCP lo fueron para la penicilina y el $50 \%$ meticilino resistentes, lo que puede representar un serio problema para salud pública.

Agradecimientos: Al CONACyT (Paraguay) por financiar el proyecto, a la Fac.Cs.Vet. (Asunción) por los fondos adjudicados, al equipo del proyecto, los es- 
tudiantes que participaron y los propietarios que permitieron trabajar con los animales.

\section{REFERENCIAS}

1. Barkema HW, Schukken YH, Zadoks RN. 2006. Invited Review: the role of cow, pathogen, and treatment regimen in the therapeutic success of bovine Staphylococcus aureus mastitis, J Dairy Sci 89: 1877-1895,

2. Blowey RW, Edmondson PW. 1995. Mastitis control in dairy herds. Farming Press, Ipswich, p. 29

3. Boireau C et al. 2018. Antimicrobial resistance in bacteria isolated from mastitis in dairy cattle in France, 2006-2016. J Dairy Sci 101: 9451-9462

4. Bonetto CC. 2014. Mastitis bovina causada por Staphylococcus coagulasa negativos. Tesis de Doctorado, Univ. Nac. de la Plata, Argentina, $206 \mathrm{p}$.

5. Clinical \& Laboratory Standards Institute. 2013. Performance standards for antimicrobial disk and dilution susceptibility tests for bacteria isolated from animals Approved standard and supplement VET01-A4 and VET01S2 (replaces M31 A3) (Fourth Edition). Wayne, PA: Clinical Laboratory Standard Institute.

6. Contreras GA, Rodríguez J M. 2011. Mastitis: comparative etiology and epidemiology. J Mammary Gland Biol Neoplasia 16: 339-356

7. Cheng $\mathbf{J}$ et al. 2019. Antimicrobial resistance profiles of 5 common bovine mastitis pathogens in large Chinese dairy herds. J Dairy Sci 102: 2416-2426.

8. Edmondson P. 2011. Blitz therapy for the eradication of Streptococcus agalactiae infections in dairy cattle. In Practice 33: 33-37.

9. Elgarch F et al. 2020. Antimicrobial susceptibility of nine udder pathogens recovered from bovine clinical mastitis milk in Europe 2016. Vet Microbiol 245: 1-13.

10. Florentín CC. 2007. Perfil de resistencia in vitro a antimicrobianos de cepas causantes de mastitis aisladas de leche cruda bovina en establecimientos de pequeña y mediana producción. Mem Inst Investig Cienc Salud 5: 19-25.

11. Gao J et al. 2012 Molecular types and antibiotic resistance of Staphylococcus aureus isolates from bovine mastitis in a single herd in China, Vet $J$ 192: 550-552.

12. Gizaw F et al. 2020. Distribution and antimicrobial resistance profile of coagulase-negative staphylococci from cattle, equipment, and personnel on dairy farm and abattoir settings. Heliyon 6 e03606: 1-11.

13. Halasa $\mathrm{T}$ et al. 2007. Economic effects of bovine mastitis and mastitis management: A review. Vet $Q 29: 18-31$.

14. Jiménez VS et al. 2020. Perfil de resistencia antimicrobiana en aislamientos de Staphylococcus sp. obtenidos de leche bovina en Colombia. Rev Argent Microbiol 52: 121130 .

15. Koneman EW, Allen S, Janda WM, Schreckenberger PC, Winn WC. 1999. Diagnóstico Microbiológico, $5^{\circ} \mathrm{Ed}$., Panamericana, Madrid, p. 528-529.
16. Kroning IS et al. 2016. Staphylococcus aureus isolated from handmade sweets: biofilm formation, enterotoxigenicity and antimicrobial resistance. Food Microbiol 58: 105-111.

17. McDougall S, Hussein H, Petrovski K. 2014. Antimicrobial resistance in Staphylococcus aureus, Streptococcus uberis and Streptococcus dysgalactiae from dairy cows with mastitis. N. Z. Vet. J. 62: 68-76.

18. National Mastitis Council. 2018. Global milk quality. procedures for collecting milk samples. On line: http:/ www.nmconline.org/sampling.htm

19. OMS. 2016. Plan de acción mundial sobre la resistencia a los antimicrobianos. Acceso. 25-8-2020. On line: https:/ www.who.int/antimicrobial-resistance/publica tions/global-action-plan/es

20. Raspanti CG et al. 2016. Prevalence and antibiotic susceptibility of coagulase-negative Staphylococcus species from bovine subclinical mastitis in dairy herds in the central region of Argentina. Rev Argent Microbiol 48: 50-56.

21. Rato MG et al. 2013. Antimicrobial resistance and molecular epidemiology of streptococci from bovine mastitis. Vet. Microbiol 161: 286-294.

22. Sánchez MP, Gutiérrez NP. 2015. Frecuencia y susceptibilidad antimicrobiana del estafilococo coagulasa negativo aislado de mastitis bovina en fincas lecheras del Tolima, Colombia. Rev Med Vet Bogota: 30: 83-93.

23. Sawant AA, Gillespie BE, Oliver SP. 2009. Antimicrobial susceptibility of coagulase negative Staphylococcus species isolated from bovine milk, Vet Microbiol 134 : $73-81$.

24. Schroeder JW. 2012. Bovine mastitis and milking management, NDSU Extention Serv 1129: 1-16.

25. Srednik ME, Abate S, Gentilini ER. 2016. Susceptibilidad antibiótica de estafilococos aislados de muestras de leche provenientes de mastitis bovina. In Vet 18: 39-44.

26. Swedberg G, Ringertz S, Skold O. 1998. Sulfonamide resistance in Streptococcus pyogenes is associated with differences in the amino acid sequence of its chromosomal dihydropteroate synthase. Antimicrob Agents Chemother 42: 1062-1067.

27. Taponen S, Nykäsenoja S, Pohjanvirta T, Pitkälä A, Pyörälä S. 2016. Species distribution and in vitro antimicrobial susceptibility of coagulase-negative staphylococci isolated from bovine mastitic milk. Acta Vet Scand 58: 1-13.

28. Vanderhaeghen W et al. 2015. Identification, typing, ecology and epidemiology of coagulase negative staphylococci associated with ruminants. Vet J 203: 44-51.

29. Zhang $\mathbf{S}$ et al. 2018. Phenotypic and genotypic characterization of antimicrobial resistance profiles in Streptococcus dysgalactiae isolated from bovine clinical mastitis in 5 provinces of China. J Dairy Sci 101: 3344-3355. 